\title{
Predictions of coronavirus incidence in New Zealand
}

\author{
Arindam Basu ${ }^{1}$ \\ ${ }^{1}$ University of Canterbury
}

April 28, 2020

\section{Coronavirus predictions in NZ and others}

New Zealand is one of the few countries that seem to have got a control over the recent covid19 outbreak.(James et al., 2020) Here we present the incidence of Coronavirus in New Zealand and some predictions as to how and when we can expect to get to a point of zero incident cases in NZ. We used the incidence package within R statistical software to create incidence objects(Kamvar et al., 2019) from data obtained from the JHU CSSE covid19 archives, and use the graphs to obtain these estimates. We used a realistic estimate of R0 2.4 for the first four weeks of the projected numbers and a series of effect reproductive numbers of 0.70 and 0.90 for the downward trend (Dietz, 1993).

\section{The incidence plot}

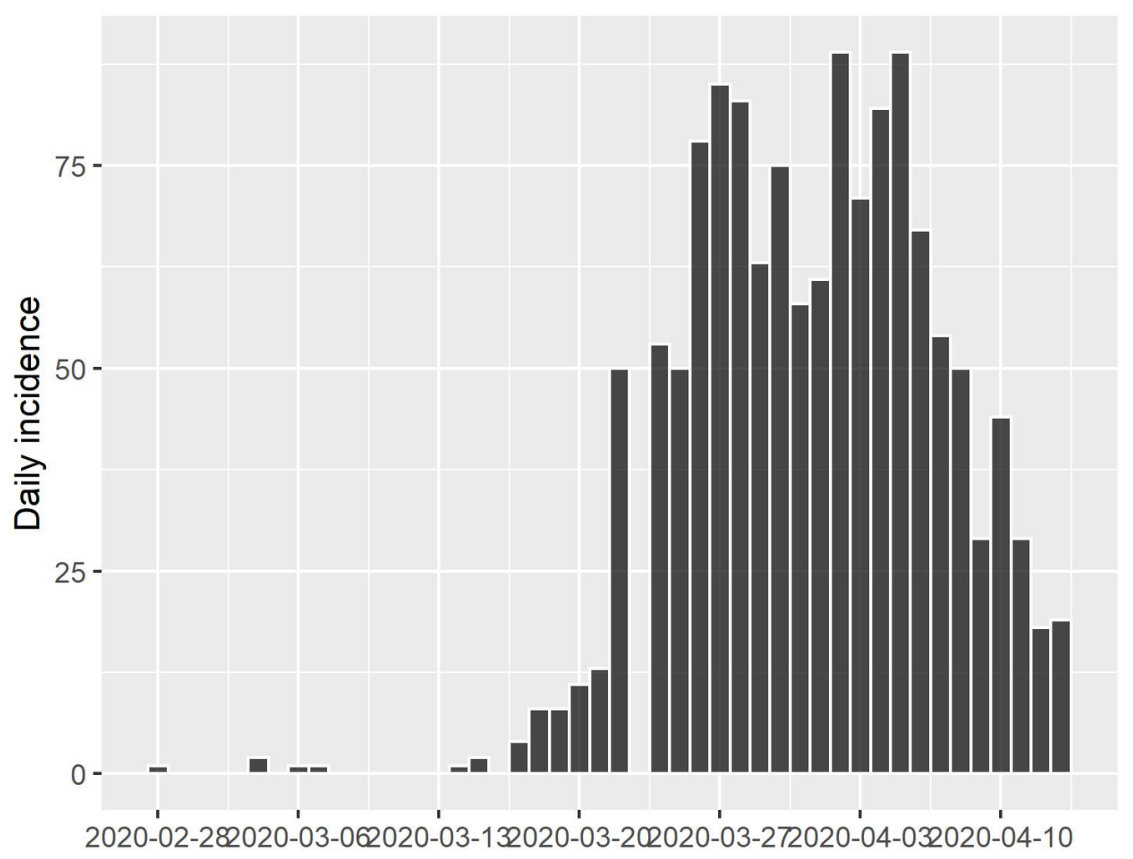

Figure 1: Incidence of covid19 cases in New Zealand, from 28th February till 10th April, 2020 


\section{Incidence plot}

This shows that the number of cases increased steadily till the first week of April and then progressively dropped. This suggests that we had reached a peak in the number of cases in New Zealand and then there has been a progressive drop in the number of new cases we continued to find despite significantly increasing the number of tests. Intuitively, this would mean that we would find more new cases even with somewhat relaxed testing criteria that the Ministry of Health used for testing (instead of tight testing criteria for overseas travel or contact with someone with history of overseas travel and physical symptoms, now the new criteria included anyone with suspicious symptoms and signs). We have fitted an optimised model to find a peak date and patterns of fitted lines on the existing data. The find_peak() function from the incidence package returns that the peak was reached around 4th April, 2020.

Next, we fitted an optimised Weibull regression model for the data using the incidence package and ran the regression model against time with no other coviariates.

\section{Results of the regression model}

In the model, we regressed log-incidence over time, splitting the dates into two halves: one before 4th April, 2020, and the ones after 4th April, 2020. Each of these graphs were exponential graphs, and had different growth rates. We obtained the following statistics from the regression model:

\begin{tabular}{lll}
\hline Parameter & Estimate & $95 \%$ confidence interval \\
\hline Daily Growth Rate before 4th April & 0.16 & $0.13,0.10$ \\
Daily growth rate after 4th April & -0.15 & $-0.22,-0.08$ \\
Doubling time of infection before 4/4/2020 & 4.31 days & $3.64,5.28$ \\
Doubling (down) time of infection after 4/4/2020 & 4.35 & $3.02,7.75$ \\
\hline
\end{tabular}

This gives us several interesting insights into the process of the growth and shrinkage of this epidemic. Prior to 4th April, the number of cases grew rapidly at the rate of doubling every nearly five days; likewise, after the peak period was reached, when the epidemic started on a downhill climb, it did so equally rapidly as evidenced over the next few days, new cases halving roughly every five days. We can graphically examine these trends. 


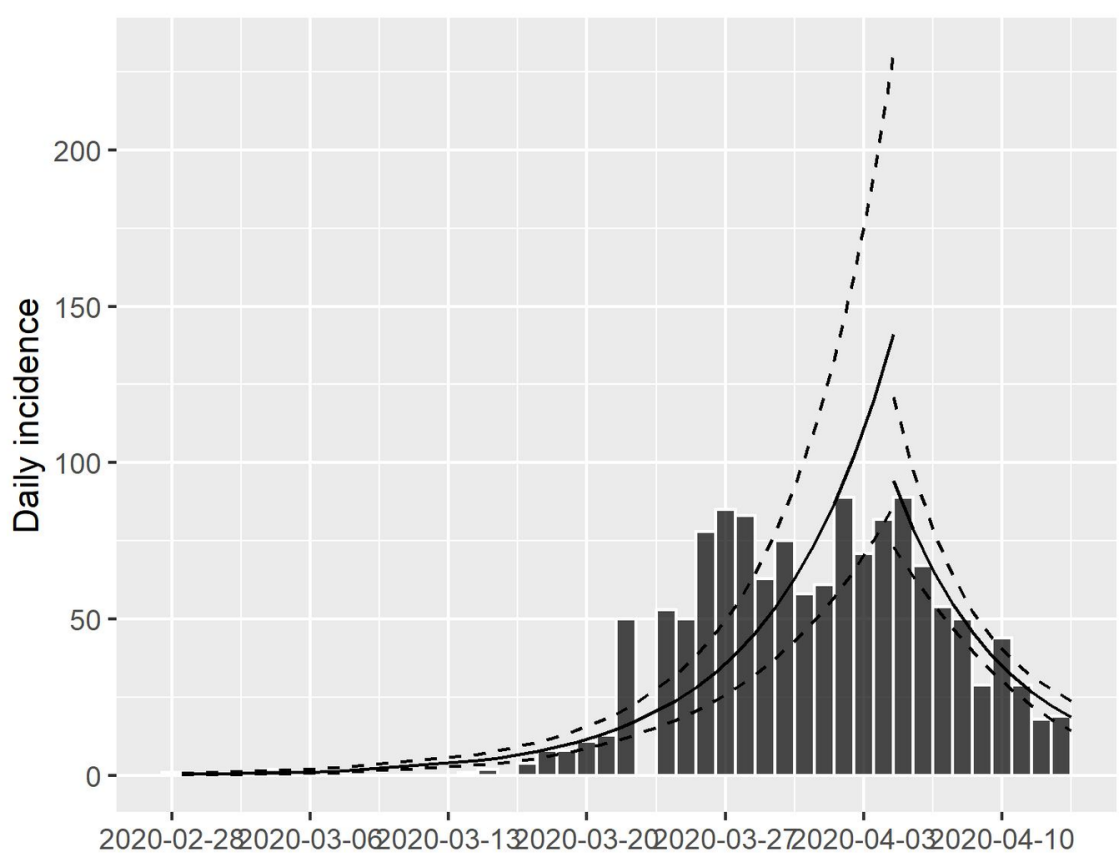

Figure 2: Regression models fitted to the incidence trend

\section{Daily incidence}

As can be seen in this graph (Figure), the trend of the cases grew rapidly till 2nd April, then there was a plateau, and then the number of cases steadily decreased starting 3rd April. In the absence of any intervention, the number of cases would have gone upward. New Zealand started "lock down" or level 4 of the intervention to "eliminate" the infection in and around 25th March, 2020. This meant that the effects to reduce the new number of cases even with increased surveillance and relaxed criteria took about 10 days to take effect. Note that this roughly or on average the time it takes an infection to manifest from first infection. This suggests that despite limitation in the number of tests being conducted, the tests were on track to identify and estimate the true number of cases.

Here are the details of these parameter estimates as of now:

\begin{tabular}{lc}
\hline Parameter & Value \\
\hline Average incident cases & 29.8 \\
standard deviation & 33.1 \\
Coefficient of variation & 0.90 \\
\hline
\end{tabular}

While the numbers continue to decline, to get to a zero incidence rate, it will be weeks before that can happen. 


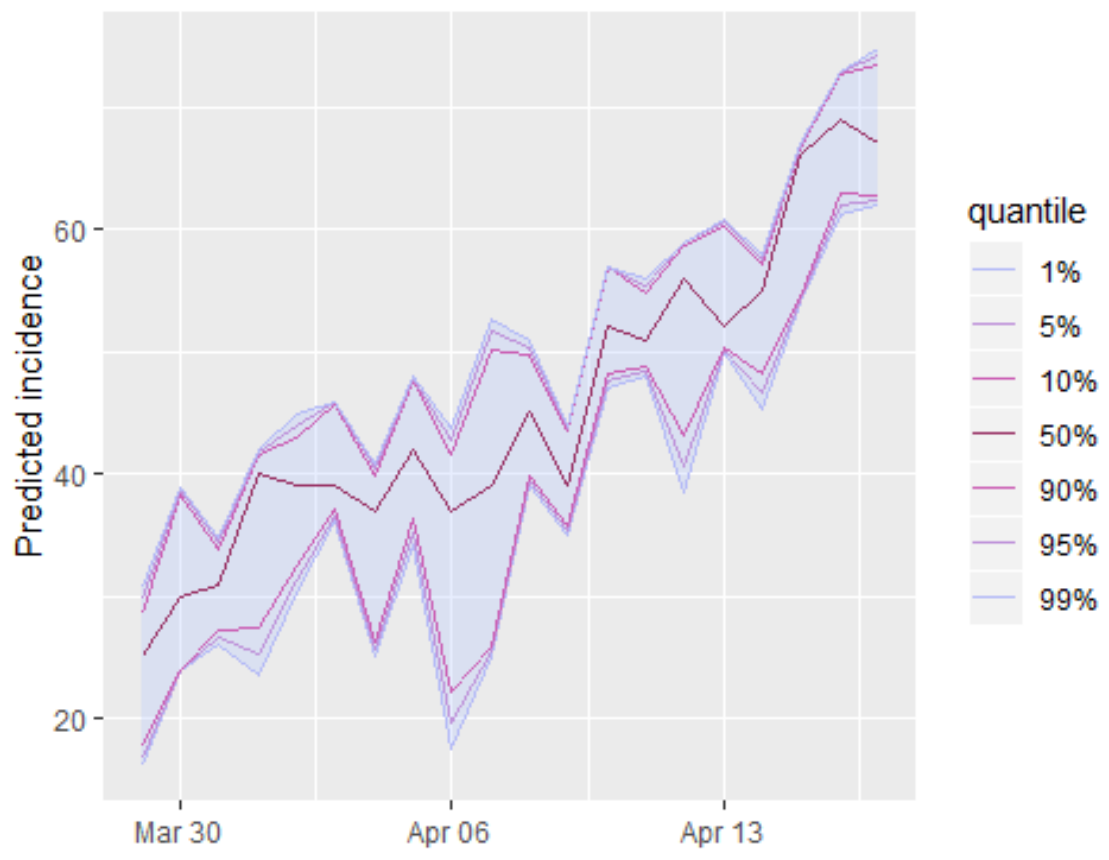

Figure 3: Projected trend in the incidence figures if the cases were to continue at the same rate in the first four weeks without any intervention such as the lock down

If we consider the trend lines based on our estimation of the possible rate of rise in the number of new cases assuming the trend of the growth in the number of cases were to continue as before, then we see from our plotting that by April 16th onward, we would see around 70 new cases everyday. But we do not see that, instead we have trickled down to around 18 cases in a day (some days may still be high, this is on an average).

\section{What happened as a result of the lock down and what can we expect?}

It is reasonable to believe that when a country goes for suppression of infection, then the aim is to bring down the effective reproduction number to below 1.0. In theory, if $100 \%$ people comply with the lock down provisions, then it should happen instantly. But that may not be the case. So, let's say enough people comply with the provisions so that the effective reproduction number drops down to around 0.90 and the infection is deemed unsustainable. Then, we should expect a slow regression to zero cases eventually. But how slow or how fast? It cannot be as rapid as the cases upswung in the first place. Let's say we study the trend of new cases for days 30 through 37 (in our case this would be something like we study from March 28 through April 6) and try to predict when we can expect the new cases will drop down to zero, so the total case count will steady with no new cases anymore.

Here is the figure: 


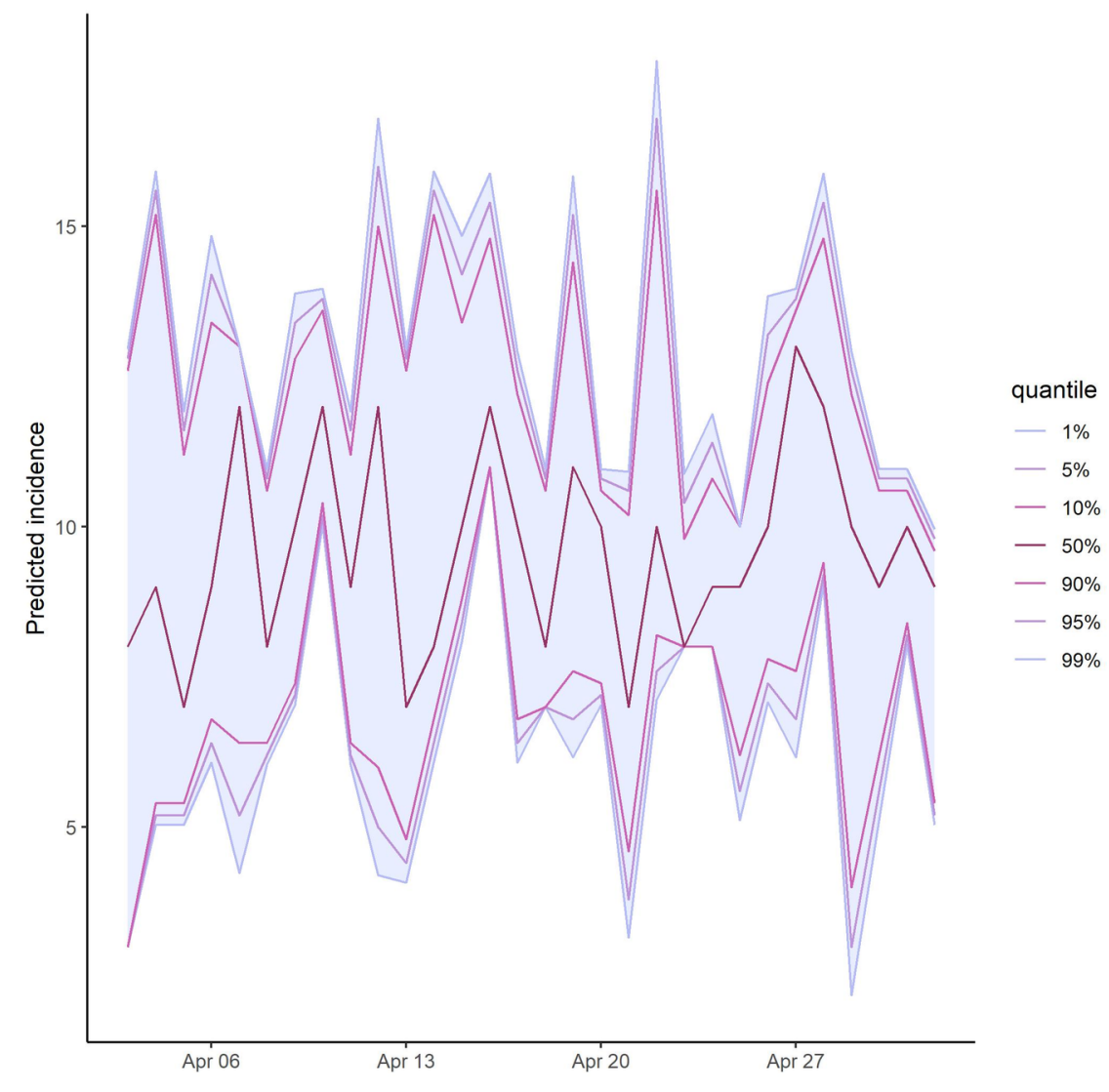

Figure 4: Projected number of cases as a result of the lock down in place given the results observed between 30 March and 5th of April 2020

\section{Drop down}

As can be seen, that countdown to zero is much slower although there does seem that most days, we will not see many new cases above 15 as does seem to be the case. We assumed an $\mathrm{R}$ of 0.70 ; what happens if we settle for a more conservative $\mathrm{R}$, such as 0.90 which may be a little more realistic?

See the next figure: 


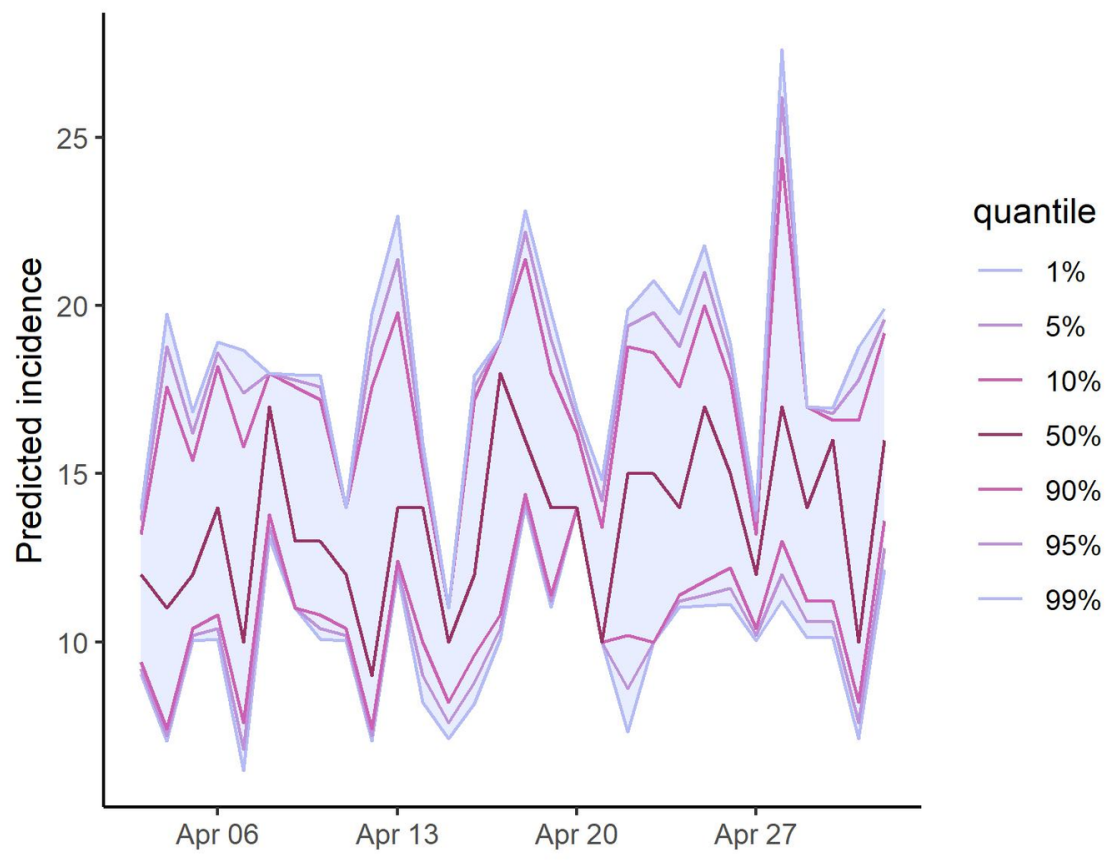

\section{Conservative estimate}

This gives us a more realistic and perhaps a little more aligned to the numbers we are seeing. The numbers are 20 and then drop down to 10 and five etc on days. We are hopeful but if you study the trajectory, you will see that we are not going to see zero new cases anytime in the middle of April even with effective reproduction number of 0.90

Hence the message is that we need to be patient and continue to practice the conditions of hand and respiratory hygiene, and maintain safe distance, even as the "lock down" is lifted. A rush at this stage is not warranted.

\section{Code and data}

Download the covid_model.Rmd and run using an Rstudio instance.

\section{Hosted file}

covid_model.rmd available at https://authorea.com/users/2191/articles/442027-predictions-ofcoronavirus-incidence-in-new-zealand

\section{References}

Suppression and Mitigation Strategies for Control of COVID-19 in New Zealand. (2020). New Zealand Medical Journal, 12.

Epidemic curves made easy using the R package incidence. (2019). F1000Research, 8, 139. https://doi. org/10.12688/f1000research.18002.1

The estimation of the basic reproduction number for infectious diseases. (1993). Stat Methods Med Res, 2(1), 23-41. https://doi.org/10.1177/096228029300200103 